\title{
A Case of Pseudoinfarction Pattern in Diabetic Ketoacidosis: A Diagnostic and Therapeutic Dilemma
}

\author{
Eliza Sharma ${ }^{\mathrm{a}}$, Suyash Dahal ${ }^{\mathrm{b}}$, Pratibha Sharma ${ }^{\mathrm{a}}$, \\ Dipesh KC Ghimire ${ }^{c}$, Sumit Dahal ${ }^{c, d}$
}

\begin{abstract}
Diabetic ketoacidosis (DKA) is regularly associated with hyperkalemia that results in well-described changes on the electrocardiogram (EKG). However, ST-segment elevations on EKG mimicking acute myocardial infarction have rarely been described in the setting of DKA. Here we present a case of a 43-year-old male with DKA who had pseudoinfarction pattern of ST-segment elevation on EKG that resolved with treatment of DKA and discuss the diagnostic and therapeutic dilemma around the condition.
\end{abstract}

Keywords: Diabetic ketoacidosis; Pseudoinfarction; Electrocardiogram

\section{Introduction}

Diabetic ketoacidosis (DKA) is characterized by ketoacidosis, hyperglycemia and dyselectrolytemia. Despite a depletion of the total body potassium content, the serum potassium concentration is usually elevated in DKA and may manifest as any dysrhythmia. A number of electrocardiogram (EKG) findings have been well described in hyperkalemia. These include peaked T-waves, widened QRS complexes with prolonged PR interval, loss of P-waves and appearance of sinusoidal waves as the QRS complexes merge with the T-waves. However, acute ST-segment elevations on EKG mimicking acute myocardial infarction (MI) have rarely been described in the setting of DKA [1-3]. Furthermore, DKA may be associated with a non-specific rise in serum troponin level and adds to the diagnostic and therapeutic dilemma. Here we describe a case of a young male with DKA who had pseudoinfarction pattern of

Manuscript submitted June 4, 2018, accepted June 23, 2018

aDepartment of Internal Medicine, Maimonides Medical Center, Brooklyn, NY, USA

bepartment of Medicine, KIST Medical College and Teaching Hospital, Lalitpur, Nepal

'Department of Medicine, Interfaith Medical Center, Brooklyn, NY, USA

${ }^{\mathrm{d} C}$ Corresponding Author: Sumit Dahal, Department of Medicine, Interfaith

Medical Center, 1545 Atlantic Avenue, Brooklyn, NY 11213, USA.

Email:sumit.dahal@gmail.com

doi: https://doi.org/10.14740/cr747w
ST-segment elevation on EKG and rise in serum troponin level that resolved with treatment of DKA.

\section{Case Report}

A 43-year-old male was out drinking beer with his friends when he started to have severe difficulty in breathing accompanied by chest pain, nausea and multiple episodes of nonbloody non-bilious vomiting. Emergency medical services (EMS) were activated and he was found to have ST-segment elevations on the cardiac monitor. He was immediately transported to our emergency room (ER) with ST-elevation myocardial infarction (STEMI) notification.

On arrival, he was still short of breath and nauseous but his chest pain had resolved. He denied any fever, cough, abdominal pain, palpitation, pain or swelling over any other part of the body. Rest of his review of symptoms was negative. His past medical history was significant for type 1 diabetes mellitus (DM) for which he was on 20 units of subcutaneous insulin glargine at bedtime and 10 units of subcutaneous insulin glulisine four times a day. He had no personal or family history of cardiac disease. He was an occasional alcohol drinker but did not smoke or use any recreational drug. On examination, he appeared anxious and was sweating. His body temperature was $36.2^{\circ} \mathrm{C}$, pulse rate was 113 beats $/ \mathrm{min}$, blood pressure was $131 / 64 \mathrm{~mm} \mathrm{Hg}$, respiratory rate was $24 / \mathrm{min}$ and oxygen saturation was $98 \%$ in room air. He had dry lips and oral mucosa with tachycardia and tachypnea without crepitation, wheezing or murmur on chest auscultation. He was awake, alert and oriented, and had no tenderness over the abdomen or any other part of the body.

An immediate EKG was done that confirmed ST-segment elevations in leads V1 and V2, and an urgent percutaneous coronary intervention (PCI) was planned (Fig. 1). In the meantime, results of his initial blood work returned and was significant for a serum troponin level of $0.5 \mathrm{ng} / \mathrm{mL}$ (normal $0.0-0.4$ $\mathrm{ng} / \mathrm{mL}$ ), serum glucose of $786 \mathrm{mg} / \mathrm{dL}$ (normal $59-140 \mathrm{mg} / \mathrm{dL}$ ), serum potassium of $8.1 \mathrm{mmol} / \mathrm{L}$ (normal $3.4-4.8 \mathrm{mmol} / \mathrm{L}$ ), serum bicarbonate of $3 \mathrm{mmol} / \mathrm{L}$ (normal $22-32 \mathrm{mmol} / \mathrm{L}$ ), serum anion gap of $38 \mathrm{mmol} / \mathrm{L}$ (normal $3-11 \mathrm{mmol} / \mathrm{L}$ ) with ketones in urine and a $\mathrm{pH}$ of 7.23 in arterial blood. Rest of his laboratory tests showed serum sodium of $117 \mathrm{mmol} / \mathrm{L}$ (normal 135 - $149 \mathrm{mmol} / \mathrm{L}$ ), serum blood urea nitrogen of $44 \mathrm{mg} / \mathrm{dL}$ (normal 7 - $21 \mathrm{mg} / \mathrm{dL}$ ), serum creatinine of $2.7 \mathrm{mg} / \mathrm{dL}$ (normal 0.5 - $1.3 \mathrm{mg} / \mathrm{dL}$ ) and a serum osmolality of $317 \mathrm{mOsm} / \mathrm{kg}$ (nor- 




Figure 1. Initial electrocardiogram showing ST-segment elevations and tall T-waves.

mal 275 - $295 \mathrm{mOsm} / \mathrm{kg}$ ). Bedside echocardiogram revealed a normal ejection fraction and absence of any significant wall motion abnormality. A diagnosis of DKA was made, and the patient was treated with intravenous fluids and insulin drip. He also received two doses of intravenous calcium gluconate $2 \mathrm{~g}$, a single dose of oral aspirin $162 \mathrm{mg}$ and an intravenous bolus of heparin 5,000 units. The planned PCI was postponed in view of the serious metabolic derangements. Interestingly, resolution of the DKA and associated metabolic derangements were accompanied by normalization of the EKG changes, and a subsequent angiography revealed patent coronaries (Fig. 2) He was discharged home after 4 days in the hospital.

\section{Discussion}

DKA is characterized by ketoacidosis and hyperglycemia, and is usually associated with different electrolyte derangements. Increased urinary and gastrointestinal loss of potassium will cause depletion in total body potassium content. However, in the milieu of acidosis and insulin deficiency, there is a shift of potassium from intracellular fluid to extracellular fluid and the initial serum potassium concentration is usually normal or elevated [4].

Hyperkalemia has a profound effect on myocardial conduction and repolarization. It may present with any dysrhyth- mia including sinus bradycardia, atrioventricular blockade of various degrees, intermittent bundle branch blocks, ventricular tachycardia and ventricular fibrillation [5]. The typically described EKG changes in hyperkalemia include peaked Twaves, widened QRS complexes with prolonged PR interval, loss of P-waves and appearance of sinusoidal waves as the QRS complexes merge with the T-waves. ST-segment elevations mimicking myocardial infarction, like in our case, have been rarely described in the setting of hyperkalemia [1, 3, 6-8]. Our patient's findings of ST-segment elevations in leads V1 and V2 were consistent with the findings of a review by Bellazzini et al who reported the anterior-septal pseudo-infarct pattern being the most common [1]. While the exact mechanism for such ST-segment elevation is unclear, it may be related to the shortening of the repolarization phase of the myocardium. This, in turn, is proposed to be due to an increased efflux of potassium by potassium-current channels on the myocyte cell membranes when the extracellular potassium concentration is high, which shortens the phase two and three of the cardiac action potentials [9]. In cases of hyperkalemia due to DKA, other coexisting metabolic abnormalities may contribute to ST-segment changes besides the direct effect of potassium on myocardium [2]. Cases of similar EKG changes in DKA with normal serum potassium level have been reported [10].

Our patient was brought by EMS with STEMI notification after being found to have ST-segment elevations on the cardiac

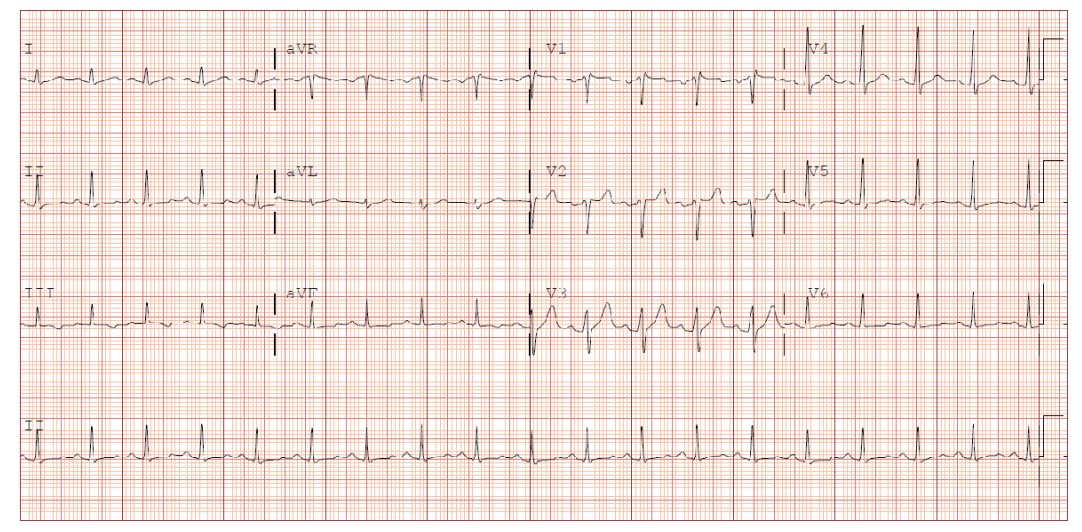

Figure 2. Follow-up electrocardiogram showing resolution of the initial changes. 
monitor. The EKG done at arrival to the hospital confirmed these findings, and he received the initial treatment accordingly. The planned PCI was postponed due to the high risks of the procedure in view of his severe metabolic derangements. As acute MI and DKA can coexist, with the former often precipitating the later, disregarding these EKG findings as being related to hyperkalemia alone can have catastrophic consequences. On the other hand, starting thrombolysis or performing urgent cardiac catheterization subjects these patients to the inherent risks of these therapies and may delay the initiation of appropriate therapy. Furthermore, DKA may be associated with a non-specific rise in serum troponin level like in our case and this adds to the diagnostic and therapeutic dilemma [11-13]. Subtle EKG clues may aid in differentiating between an actual infarction and a pseudoinfarction of hyperkalemia. While tall T-waves may be seen in both hyperkalemia and hyperacute myocardial ischemia, they tend to be tall, narrow and tented with a short or normal QTc interval in hyperkalemia as opposed to being tall and broad-based usually with long QTc interval in myocardial injury [8].

In conclusion, ST-segment elevations on EKG in DKA present a diagnostic and therapeutic dilemma. This is all the more relevant with the current emphasis on reducing the doorto-needle times for thrombolysis or primary PCI to reduce the morbidity and mortality associated with coronary artery diseases. Physicians need to be aware of this rare manifestation of DKA so as to differentiate it from a true MI and avoid unwarranted treatments and their complications.

\section{Conflict of Interest}

The authors declare that there is no conflict of interest regarding the publication of this paper.

\section{References}

1. Bellazzini MA, Meyer T. Pseudo-myocardial infarction in diabetic ketoacidosis with hyperkalemia. J Emerg Med. 2010;39(4):e139-141.

2. Sweterlitsch EM, Murphy GW. Acute electrocardio- graphic pseudoinfarction pattern in the setting of diabetic ketoacidosis and severe hyperkalemia. Am Heart J. 1996;132(5):1086-1089.

3. Ziakas A, Basagiannis C, Stiliadis I. Pseudoinfarction pattern in a patient with hyperkalemia, diabetic ketoacidosis and normal coronary vessels: a case report. J Med Case Rep. 2010;4:115.

4. Adrogue HJ, Lederer ED, Suki WN, Eknoyan G. Determinants of plasma potassium levels in diabetic ketoacidosis. Medicine (Baltimore). 1986;65(3):163-172.

5. Alfonzo AV, Isles C, Geddes C, Deighan C. Potassium disorders - clinical spectrum and emergency management. Resuscitation. 2006;70(1):10-25.

6. Peerbhai S, Masha L, DaSilva-DeAbreu A, Dhoble A. Hyperkalemia masked by pseudo-stemi infarct pattern and cardiac arrest. Int J Emerg Med. 2017;10(1):3.

7. Pothiawala SE. Hyperkalemia induced pseudo-myocardial infarction in septic shock. J Postgrad Med. 2014;60(3):338-340.

8. Wang K. Images in clinical medicine. "Pseudoinfarction" pattern due to hyperkalemia. N Engl J Med. 2004;351(6):593.

9. Roden DM, Lazzara R, Rosen M, Schwartz PJ, Towbin J, Vincent GM. Multiple mechanisms in the long-QT syndrome. Current knowledge, gaps, and future directions. The SADS Foundation Task Force on LQTS. Circulation. 1996;94(8):1996-2012.

10. Aksakal E, Duman H, Ulus T, Bayram E. Acute inferior pseudoinfarction pattern in a patient with normokalemia and diabetic ketoacidosis. Am J Emerg Med. 2009;27(2):251 e253-255.

11. Atabek ME, Pirgon O, Oran B, Erkul I, Kurtoglu S. Increased cardiac troponin I concentration in diabetic ketoacidosis. J Pediatr Endocrinol Metab. 2004;17(8):10771082.

12. Geddes J, Deans KA, Cormack A, Motherwell D, Paterson K, O'Reilly DS, Fisher BM. Cardiac troponin I concentrations in people presenting with diabetic ketoacidosis. Ann Clin Biochem. 2007;44(Pt 4):391-393.

13. Abdo AS, Geraci SA. Significance of elevated cardiac troponin I in patients with diabetic ketoacidosis. J Miss State Med Assoc. 2013;54(5):127-130. 